\title{
Aberrantly sustained PAX5 expression in plasma cell differentiation is a frequent feature in lymphoplasmacytic lymphoma but not marginal zone lymphoma in bone marrow
}

\author{
Yen-Chun Liu • Yifang Liu • Daniel M. Knowles • \\ Attilio Orazi • Wayne Tam
}

Received: 14 March 2013 / Accepted: 23 July 2013 /Published online: 8 August 2013

(C) Springer-Verlag Berlin Heidelberg 2013

\begin{abstract}
Subclassification of low-grade B cell lymphomas with plasmacytic differentiation can be a diagnostic challenge, especially in the bone marrow (BM). The major differential diagnoses include marginal zone lymphoma (MZL) and lymphoplasmacytic lymphoma (LPL). We hypothesize that the lymphoplasmacytic populations present in these two B cell lymphoma subtypes may differ in their spectrum of plasma cell differentiation. Plasma cell differentiation is gradual and phenotypically defined. PRDM1/Blimp-1 expression is initiated in B cells that are destined to become plasma cells, and precedes CD138 expression. We utilized PRDM1/PAX5 and CD138/PAX5 double immunohistochemistry to characterize and assess the differences of the plasmacytic populations in a series of MZLs, with or without plasmacytic differentiation, and LPLs. A higher percentage of CD138+ or PRDM1+ cells coexpressed PAX5 in LPL than in MZL without plasmacytic differentiation ( $p=0.013$ and 0.06 , respectively, two-tailed Mann-Whitney test). The percentage of CD138+ cells with PAX5 positivity also tends to be higher in LPL compared with MZL with plasmacytic differentiation ( $p=0.07$, two-tailed Mann-Whitney test). A similar percentage of PRDM1+ cells coexpressing PAX5 were noted in LPLs and MZLs with plasmacytic differentiation. These results demonstrate that PAX5 is aberrantly sustained in a subset of CD138+ cells in many LPLs and suggest that PAX5 deregulation is a frequent feature of LPL. These findings may provide a novel area of investigation into the underlying pathogenesis of LPL.
\end{abstract}

Y.-C. Liu • Y. Liu • D. M. Knowles · A. Orazi • W. Tam $(\square)$ Department of Pathology and Laboratory Medicine, Weill Cornell Medical College, 525 East 68th Street, Starr 715,

New York, NY, USA

e-mail:wtam@med.cornell.edu
Keywords PAX5 · Plasma cell · Lymphoplasmacytic lymphoma $\cdot$ Marginal zone lymphoma

\section{Introduction}

Lymphoplasmacytic lymphoma (LPL) is a distinct neoplasm composed of small B lymphocytes, plasmacytoid lymphocytes, and plasma cells. The diagnosis can only be made when the lesion does not fulfill the criteria for any of the other small B-cell lymphoid neoplasms that may also have plasmacytic differentiation [1]. The distinction between LPL and other small B-cell lymphoid neoplasms such as marginal zone lymphoma (MZL), especially when the latter exhibits plasmacytic differentiation, is not always clear-cut. The significant overlap in morphology and immunophenotype among these disease entities and the lack of disease-defining features in LPL contribute to the diagnostic challenges.

A central and distinguishing feature of LPL is its consistent propensity for the neoplastic B cells to differentiate into plasma cells. Plasma cell differentiation is a tightly regulated process orchestrated by a panel of transcription factors [2-6], among which are PAX5 and PRDM1/Blimp-1. PAX5, a critical transcription factor for B cell development, is known to contribute to the unique $B$ cell identity and function by directly activating and repressing target genes. These targets include cell surface receptors and the intracellular signaling transducers that are essential for lineage commitment [7, 8]. PRDM1/Blimp-1 has long been considered to be the master regulator in plasma cell differentiation through multiple mechanisms, including repression of PAX5 [9]. The process of plasma cell differentiation can be more precisely divided into a PRDM1/Blimp-1-independent stage and a PRDM1/Blimp1 -dependent stage $[3,10]$. The former is signaled primarily by downregulation of PAX5 which can drive B cells to a pre- 
plasmablast stage; while the latter is characterized by upregulation of PRDM1/Blimp-1, which further represses PAX5 and promotes full terminal differentiation into mature plasma cells.

Recently, through whole-genome sequencing and Sanger sequencing of an expanded cohort of samples, MYD88 L265P somatic mutation was found present in the vast majority of the LPL cases while only present in a relatively small number of MZL samples [11]. Further studies suggest the association between MYD88 signaling and NF-kB activation through in vitro inhibition study [11]. It is unclear at this point how MYD88 mutation alters the process of plasma cell differentiation and results in the clinical presentation of LPL, though it is believed that the deregulated expression of critical transcription factors has contributed to the pathogenesis.

With PAX5 being a critical regulator in plasma cell differentiation, it is intriguing to hypothesize that PAX5 expression may be perturbed during this process and potentially play a role in its pathogenesis of LPL. Thus, we evaluated the expression of PAX5 in PRDM1-positive cells and CD138positive cells in a collection of bone marrow (BM) samples involved by LPL. Since the expression of PRDM1/Blimp-1 is known to precede CD138 expression in plasma cell development $[2,12]$, we were able to document the dynamic course of PAX5 expression during plasma cell differentiation in this disease entity. We also evaluated PAX5 expression in BM samples involved by MZL with plasmacytic differentiation, a morphologically similar entity. We aimed to examine the possibility that abnormal PAX5 expression during plasma cell differentiation is a pathogonomic finding for lymphoplasmacytic lymphoma.

\section{Materials and methods}

\section{Samples}

Bouin-fixed, paraffin-embedded bone marrow biopsy specimens of LPL $(n=23)$ and MZL $(n=16)$ were retrieved from the archives of the Department of Pathology and Laboratory Medicine at Weill Cornell Medical College according to the protocol approved by the Institutional Review Board. All samples were reviewed and classified according to the 2008 World Health Organization criteria [1]. The delineation of the subclassification is further described in the results section and summarized in Table 2. In addition, sections of normal tonsil were included as controls.

Immunophenotypic analysis

Five-micrometer-thick formalin-fixed and paraffin-embedded tissue sections were used for immunohistochemical analyses. Double immunohistochemical stainings of PAX5/PRDM1,
PAX5/CD138, and BCL1/CD138 were performed by staining each dual combination sequentially using the Bond III Autostainer (Leica Microsystems, IL). Tissue sections were first baked and deparaffinized. For both first and second antibody stainings of each combination, sections were subjected to antigen retrieval, followed by sequential incubation with endogenous enzyme block, primary antibody, secondary antibody, tertiary antibody, and chromogen. After the second antibody staining, the slides were counterstained, dehydrated in $100 \%$ ethanol, and mounted in Cytoseal ${ }^{\mathrm{TM}}$ XYL (Richard-Allan Scientific). The specifics of each step are indicated in Table 1. Technically suboptimal stained sections were excluded from the analyses. All the MZL cases were subjected to single immunohistochemical stains including CD138 (clone B-A38; Serotec, Raleigh, NC, USA), $k$, and $\lambda$ (Dako Cytomation, Glostrup, Denmark) to determine the presence of plasmacytic differentiation by light chain restriction.

Interpretation of immunohistochemistry

The number of CD138-positive cells and the number of CD138/PAX5 double positive cells were counted in ten different high power fields. The percentage of CD138-positive cells coexpressing PAX5 was subsequently calculated. The percentage of PRDM1-positive cells coexpressing PAX5 was estimated using the same methodology (Fig. 1). Due to the inherent limitation of the PRDM1 immunohistochemical staining in the Bouin-fixed and decalcified BM biopsy section, the intensity of the PRDM1 staining was lower when compared with other cytoplasmic stainings in the BM biopsy sections.

\section{Statistical analyses}

Non-parametric Fisher's exact test and Mann-Whitney test were used for statistical analyses. All the $p$ values were based on two-tailed tests.

\section{Results}

Summary of the histologic and clinical features of the LPL and MZL cases

Twenty-three BM specimens involved by LPL were retrieved over a 3-year period. All the LPL cases had a corresponding IgM monoclonal gammopathy ranging from 1.1 to $3.5 \mathrm{~g} / \mathrm{dl}$. The patients ranged in age from 43 to 94 years with a median of 70 years and a male to female ratio of $2.3: 1$. The involvement of LPL in these samples varied from $10 \%$ to more than $95 \%$ (median $50 \%$ ). The LPL cases included in the study showed variable lymphoma infiltration patterns including interstitial, paratrabecular, focally nodular, diffuse, and mixed. 


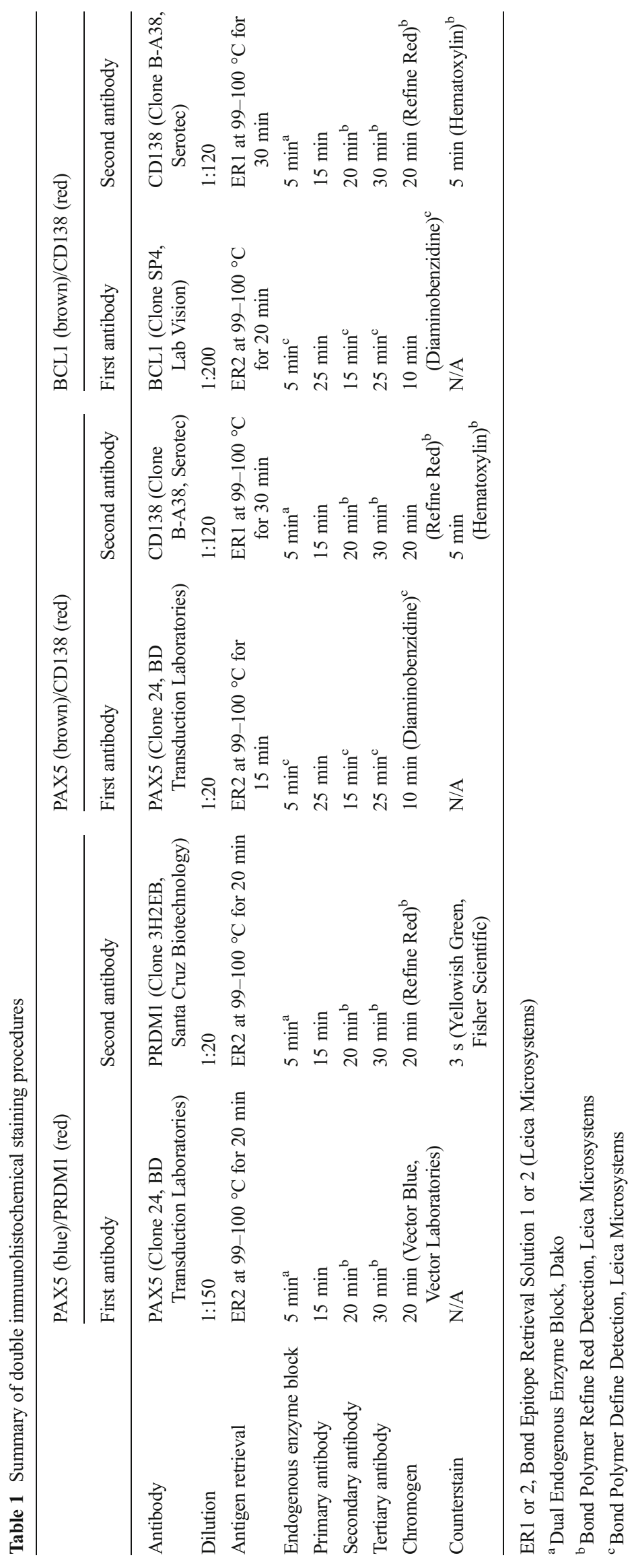




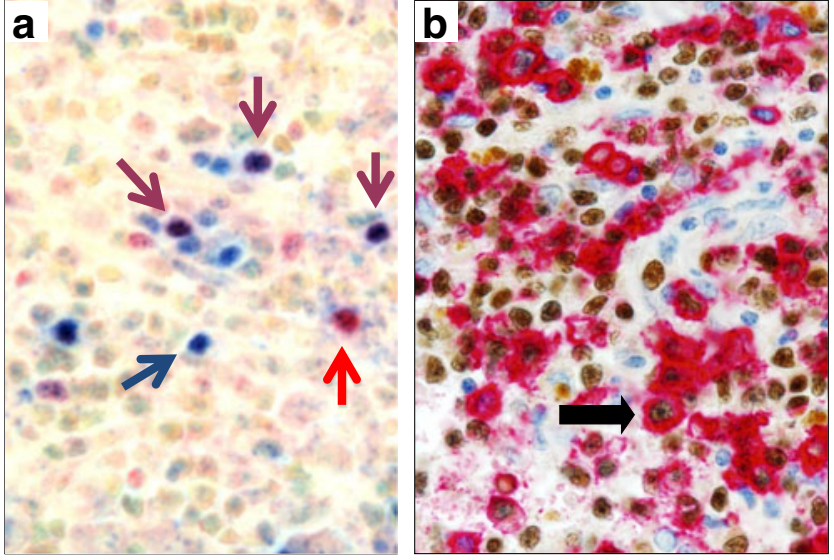

Fig. 1 Double immunohistochemical stains demonstrate the presence of PAX5+ PRDM1+ and CD138+ PRDM1+ double positive cells (a). PRDM1-positive cells showed red nuclear staining (red arrow), while PAX5-positive cells showed blue nuclear staining (blue arrow). Cells double positive for PRDM1 and PAX5 demonstrated purple nuclear staining (purple arrow). b CD138-positive cells showed red membranous staining, while PAX5-positive cells showed brown nuclear staining. Cells double positive for CD138 and PAX5 are indicated (arrowhead)

Sixteen BM specimens involved by MZL from patients who had extramedullary lesions diagnosed with MZL were retrieved over a 6-year period. Among them, four specimens had corresponding splenic B-cell marginal zone lymphoma and six specimens had corresponding extranodal marginal zone lymphoma of mucosa-associated lymphoid tissue (MALT lymphoma). Six of the BM specimens involved by MZL had corresponding MZL diagnoses that could not be definitively subclassified. The patients in the MZL group ranged in age from 43 to 81 years with a median of 61.5 years and a male to female ratio of 1:2.2. The involvement by MZL varied from $20 \%$ to more than $95 \%$ (median $70 \%$ ). Similar to the LPL samples, the MZL samples demonstrated various infiltration patterns including interstitial, paratrabecular, diffuse, and mixed pattern. Based on CD138 and the cytoplasmic light chain expression by immunohistochemistry, we further divided the MZL cases into two groups: MZL with plasmacytic differentiation $(n=9)$ and MZL without plasmacytic differentiation $(n=7)$. The age distribution and male to female ratio between the two subgroups were similar. The clinical and histologic features for all the cases are summarized in Table 2.

\section{Aberrantly sustained PAX5 expression in LPL}

All the LPL and MZL BM specimens were subjected to CD138/PAX5 double immunohistochemistry (Fig. 1). We identified CD138-positive PAX5-positive cells in 19 out of 22 BM specimens involved by LPL, while similar cells were only identified in seven out of $16 \mathrm{BM}$ specimens involved by MZL ( $p=0.0116$, Fisher's exact test.). For the entire LPL group, the percentage of CD138-positive cells coexpressing PAX5 ranged from 0 to $69.6 \%$ (median, $2.0 \%$ ). For MZL, the percentage of CD138-positive cells coexpressing PAX5 ranged from 0 to $5.1 \%$, except for an outlier of $24.8 \%$ (median, $0 ; p=0.007$, two-tailed Mann-Whitney test).

We further divided the MZL cases into MZL with plasmacytic differentiation and MZL without plasmacytic differentiation. The CD138-positive plasma cells in the latter group most likely represented bystander reactive plasma cells and could be used as a control group. The CD138-positive plasma cells in the former group were considered to be part of the neoplastic process of MZL.

In the group of MZLs without plasmacytic differentiation, coexpression of PAX5 in the CD138-positive cells was found in three of seven cases ( $p=0.0377$ vs LPL, Fisher's exact test). The percentage of CD138+ cells co-expressing PAX5 in this group ranged from 0 to $1.3 \%$ (median, 0 ). Coexpression of PAX5 in the neoplastic CD138-positive cells in the group of MZLs with plasmacytic differentiation was noted in four of nine specimens ( $p=0.0272$ vs LPL, Fisher's exact test). The percentage of CD138-positive cells coexpressing PAX5 ranged from 0 to $24.8 \%$ with the median being 0 . The difference in percentage of CD138-positive cells coexpressing PAX5 was statistically significant between LPL and MZL without plasmacytic differentiation ( $p=0.013$, two-tailed Mann-Whitney test) and the difference was marginally significant between LPL and MZL with plasmacytic differentiation ( $p=0.07$, two-tailed Mann-Whitney test; Fig. 2).

PRDM1 expression begins in early plasma cell differentiation and precedes the expression of CD138. We evaluated PAX5 coexpression in PRDM1-positive cells to determine PAX5 expression along the plasma cell differentiation process before the terminal stage. All 43 LPL and MZL BM specimens underwent PRDM1/PAX5 double immunohistochemistry. We identified PRDM1-positive, PAX5-positive cells in 18 of 19 LPL specimens and five of six BM specimens involved by MZL with plasmacytic differentiation ( $p=0.43$, Fisher's exact test). The percentage of PRDM1-positive cells coexpressing PAX5 ranged from 0 to $26.7 \%$ in the LPL group (median, $8.0 \%$ ) and 0 to $29.0 \%$ in MZLs with plasmacytic differentiation (median, $5.0 \%$ ). In MZL without plasmacytic differentiation, weakly stained PRDM1/PAX5 double positive cells were found in two of five cases ( $p=0.0184$ vs LPL, Fisher's exact test) with the percentage being 2.6 and $19.0 \%$ (median is 0 for the entire subgroup). There was no significant difference in the percentage of PRDM1-positive cells coexpressing PAX 5 between LPL and MZL with plasmacytic differentiation $(p=0.642$, two-tailed Mann-Whitney test), or between MZL with or without plasmacytic differentiation ( $p=0.247$, two-tailed Mann-Whitney test). However, the difference between LPL and MZL without plasmacytic differentiation was marginally significant $(p=0.06$, two-tailed Mann-Whitney test; Fig. 3).

It is noted that three of the MZL cases with plasmacytic differentiation included in our study had monoclonal IgM protein that exceeded $2.5 \mathrm{~g} / \mathrm{dl}$; one of them had a corresponding 
Table 2 Summary of clinical and laboratory features in the lymphoplasmacytic lymphoma and marginal zone lymphoma cases
$N / A$ cases with no available information regarding immunofixation electrophoresis, MZL marginal zone lymphoma, $P D$ plasmacytic differentiation, $M A L T$ extranodal marginal zone lymphoma of mucosa-associated lymphoid tissue, $S M Z L$ splenic marginal zone lymphoma

\begin{tabular}{|c|c|c|c|c|c|c|c|}
\hline & \multirow[t]{2}{*}{ Age } & \multirow[t]{2}{*}{ Gender } & \multirow[t]{2}{*}{ Diagnosis } & \multirow[t]{2}{*}{ Involvement } & \multicolumn{2}{|l|}{ M-spike } & \multirow[t]{2}{*}{ Note } \\
\hline & & & & & & $(\mathrm{g} / \mathrm{dl})$ & \\
\hline 1 & 76 & M & LPL & $60 \%$ & $\mathrm{IgMk}$ & 2.1 & \\
\hline 2 & 64 & M & LPL & $30 \%$ & $\operatorname{IgMk}$ & 1.6 & \\
\hline 3 & 79 & $\mathrm{~F}$ & LPL & $40 \%$ & $\operatorname{IgMk}$ & 2 & \\
\hline 4 & 59 & M & LPL & $20 \%$ & IgMk & 1.6 & Neuropathy \\
\hline 5 & 64 & M & LPL & $20 \%$ & IgMl & 1.7 & \\
\hline 6 & 67 & M & LPL & $70 \%$ & IgMl & 2.3 & \\
\hline 7 & 83 & M & LPL & $70 \%$ & IgMl & 1.7 & \\
\hline 8 & 73 & $\mathrm{~F}$ & LPL & $>95 \%$ & IgMk & 2.6 & \\
\hline 9 & 78 & M & LPL & $>90 \%$ & $\operatorname{IgMk}$ & 3.4 & \\
\hline 10 & 61 & $\mathrm{~F}$ & LPL & $30 \%$ & IgMk & 1.1 & \\
\hline 11 & 61 & M & LPL & $70 \%$ & IgMk & 3 & \\
\hline 12 & 72 & M & LPL & $25 \%$ & $\operatorname{IgMk}$ & 1.2 & \\
\hline 13 & 54 & M & LPL & $10 \%$ & $\operatorname{IgMk}$ & 2.6 & \\
\hline 14 & 63 & M & LPL & $30 \%$ & $\operatorname{IgMk}$ & 1.3 & \\
\hline 15 & 72 & M & LPL & $40 \%$ & IgMl & 1.5 & \\
\hline 16 & 72 & M & LPL & $50 \%$ & $\operatorname{IgMk}$ & 2.8 & \\
\hline 17 & 71 & M & LPL & $20 \%$ & IgMk & 1.4 & \\
\hline 18 & 94 & M & LPL & $20 \%$ & $\operatorname{IgMk}$ & 1.3 & \\
\hline 19 & 54 & $\mathrm{~F}$ & LPL & $70 \%$ & IgMk & 3.5 & \\
\hline 20 & 70 & $\mathrm{~F}$ & LPL & $90 \%$ & IgMl & 2.2 & \\
\hline 21 & 59 & M & LPL & $80 \%$ & $\operatorname{IgMk}$ & 2.4 & Neuropathy \\
\hline 22 & 71 & $\mathrm{~F}$ & LPL & $80 \%$ & IgMk & 1.5 & \\
\hline 23 & 43 & $\mathrm{~F}$ & LPL & $80 \%$ & $\mathrm{IgMk}$ & 2 & \\
\hline 24 & 55 & $\mathrm{~F}$ & MZL with PD & $30 \%$ & $\operatorname{IgMk}$ & 0.7 & MALT \\
\hline 25 & 67 & $\mathrm{~F}$ & MZL with PD & $30 \%$ & IgGk & 4.4 & MZL \\
\hline 26 & 61 & M & MZL with PD & $20 \%$ & IgMk & 0.05 & SMZL \\
\hline 27 & 81 & $\mathrm{~F}$ & MZL with PD & $70 \%$ & IgGk & 0.05 & SMZL \\
\hline 28 & 43 & $\mathrm{~F}$ & MZL with PD & $30 \%$ & $\mathrm{~N} / \mathrm{A}$ & & MZL \\
\hline 29 & 46 & $\mathrm{~F}$ & MZL with PD & $80-90 \%$ & N/A & & MALT \\
\hline 30 & 60 & M & MZL with PD & $>95 \%$ & IgMl & 2.7 & MZL \\
\hline 31 & 61 & M & MZL with PD & $70-80 \%$ & $\operatorname{IgMk}$ & 2.8 & MZL \\
\hline 32 & 75 & $\mathrm{~F}$ & MZL with PD & $90 \%$ & IgMl & 6.12 & MALT \\
\hline 33 & 73 & M & MZL without PD & $80 \%$ & No peak & & MZL \\
\hline 34 & 59 & $\mathrm{~F}$ & MZL without PD & $20 \%$ & N/A & & MALT \\
\hline 35 & 73 & $\mathrm{~F}$ & MZL without PD & $50 \%$ & No peak & & MALT \\
\hline 36 & 74 & M & MZL without PD & $80 \%$ & No peak & & MALT \\
\hline 37 & 73 & $\mathrm{~F}$ & MZL without PD & $80-90 \%$ & N/A & & MZL \\
\hline 38 & 62 & $\mathrm{~F}$ & MZL without PD & $70 \%$ & No peak & & SMZL \\
\hline 39 & 55 & $\mathrm{~F}$ & MZL without PD & $60 \%$ & No peak & & SMZL \\
\hline
\end{tabular}

diagnosis of extranodal marginal zone lymphoma of mucosaassociated lymphoid tissue (MALT lymphoma) while the other two had corresponding MZL diagnoses that could not be definitively subclassified (Table 2). The high monoclonal spike coupled with the rather extensive marrow infiltration raises the possibility that these cases might actually represent LPL with extramedullary involvement. CD138+ PAX5+ cells were found in two of three cases and the percentage of CD138+ cells coexpressing PAX5 in this group ranged from 0 to $5.1 \%$ (median, $0.5 \%$ ). In addition, PRDM1+ PAX5+ cells were also found in two of three cases and the percentage of PRDM1+ cells coexpressing PAX5 ranged from 0 to $14.3 \%$ (median, $1.8 \%$ ). When these three cases were treated as a separate category, there was no significant difference in the percentage of CD138+ 
Fig. 2 A higher percentage of CD138-positive cells coexpressing PAX5 was identified in LPL when compared with MZL without plasmacytic differentiation (two-tailed $p=0.013)$ and MZL with plasmacytic differentiation (twotailed $p=0.07$ )

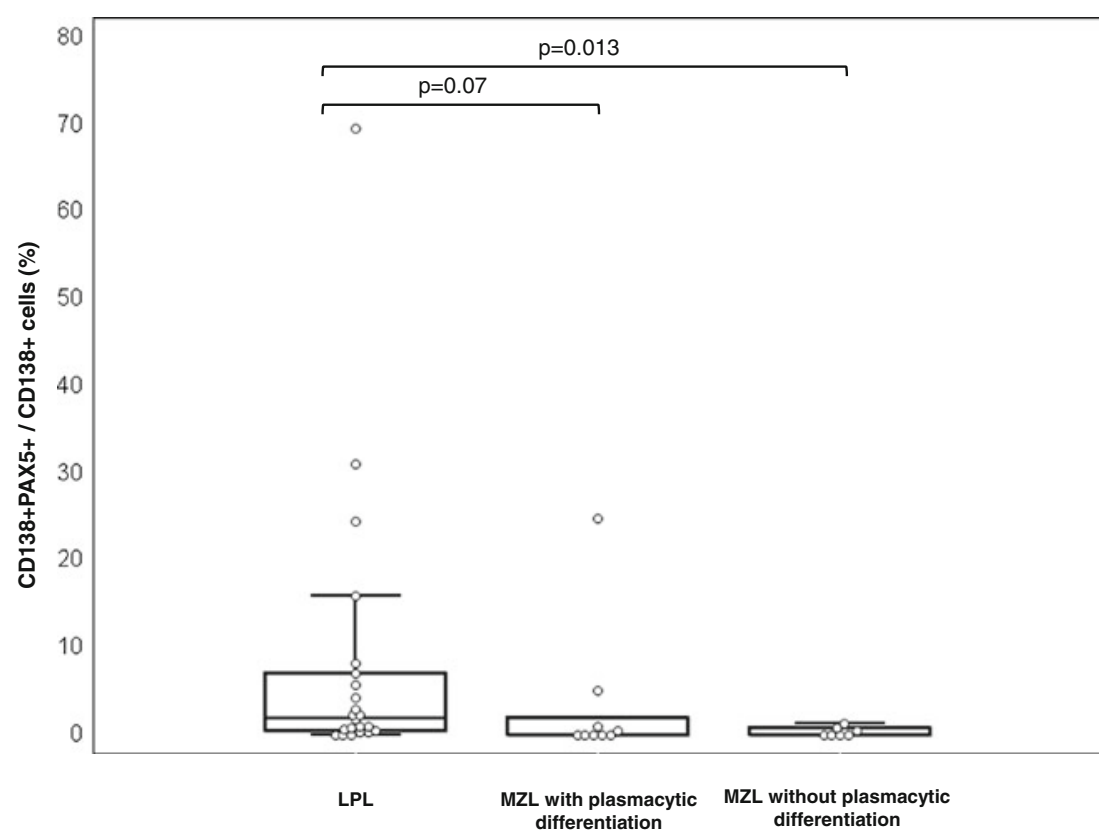

cells coexpressing PAX5 or the percentage of PRDM1+ cells coexpressing PAX5 between this category and LPL or this category and other MZL cases with plasmacytic differentiation. Comparing LPL with MZL with plasmacytic differentiation excluding the three cases still demonstrated a trend of higher percentage of CD138-positive cells coexpressing PAX5 in LPL ( $p=0.088$, two-tailed Mann-Whitney test) and revealed no significant difference in the percentage of PRDM1+ cells coexpressing PAX5 ( $p=0.787$, two-tailed Mann-Whitney test), similar to LPL vs MZL with plasmacytic differentiation including the three cases. If these three cases were incorporated into the LPL category instead of MZL for statistical analysis, the conclusions previously stated remained valid.

The distinction among disease entities such as IgM multiple myeloma, multiple myeloma with CD20 expression, and lymphoplasmacytic lymphoma is critical for clinical management as well as research aiming to elucidate the underline pathogenesis for these diseases. The rare entity of IgM multiple myeloma has previously been defined; the vast majority of these cases have cyclin D1 expression by immunohistochemical staining and are characterized by $\mathrm{t}(11 ; 14)$ through cytogenetics studies $[13,14]$. The neoplastic plasma cells in this
Fig. 3 A higher percentage of PRDM1-positive cells coexpressing PAX5 was identified in LPL when compared with MZL without plasmacytic differentiation (two-tailed $p=0.06$ ). There is no significant difference in percentage of PRDM1-positive cells coexpressing PAX5 between LPL and MZL with plasmacytic differentiation

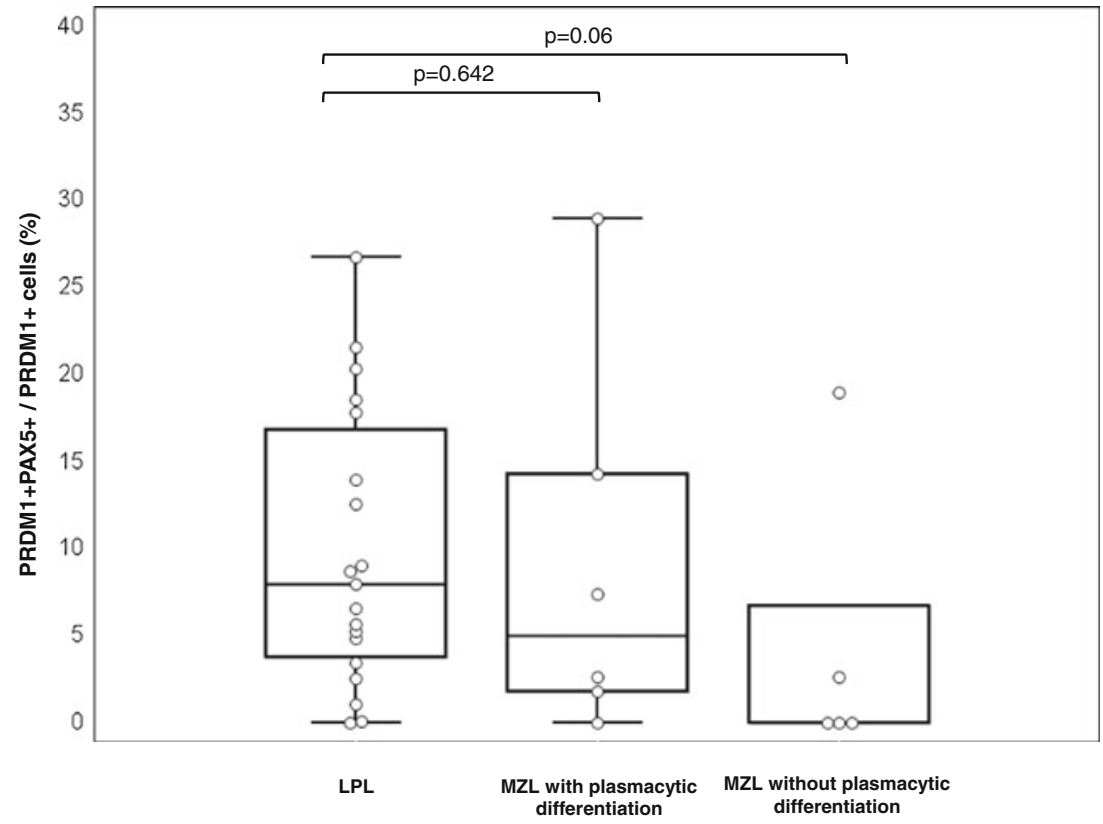


rare entity were reported to be negative for CD20, CD56, and CD117 [14]. Multiple myeloma with CD20 expression, on the other hand, shows a slightly different cytomorphology: small mature plasma cells with less cytoplasm, and associates with the $t(11 ; 14)$ genotype $[15]$, a recurrent abnormality that associates with nuclear cyclinD1 expression $[16,17]$. Separate report demonstrated concordant PAX5 expression in myelomas with CD20 expression [18]. The rare entity of IgM myeloma has previously been defined as a monoclonal plasma cell proliferative disorder with a serum IgM monoclonal protein, greater than $10 \%$ of plasma cells on bone marrow biopsy, and the presence of lytic bone lesions related to the underlying plasma cell disorder and/or $\mathrm{t}(11 ; 14)$ on fluorescent in situ hybridization. None of the LPL cases included in our case series had osteolytic lesions and none of them fulfilled the proposed diagnostic criteria for IgM multiple myeloma. CD138/cyclin D1 double immunohistochemistry was also performed on the majority of LPL cases $(n=15)$, including all the BM specimens with more than $5 \%$ of CD138-positive cells coexpressing PAX5. None of these cases showed cyclin D1 expression (data not shown) and thus are unlikely to fall into the category of myeloma with CD20 expression.

Presence of PRDM1/PAX5 double positive cells in reactive germinal centers

The presence of cells double positive for PRDM1 and PAX5 has been reported in reactive germinal centers using immunohistochemistry performed on frozen sections [19]. We confirmed the presence of PRDM1 and PAX5-double positive cells (approximately $1-2 \%$ of germinal center B cells) by immunoperoxidase staining on formalin-fixed, paraffin-embedded tissue sections of normal tonsils (Fig. 4.) Double positive cells for CD138 and

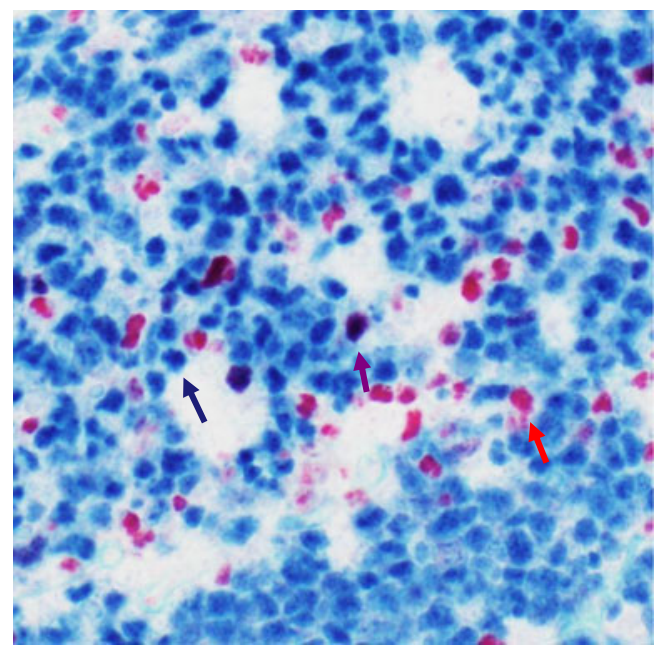

Fig. 4 PRDM1+ and PAX5+ double positive cells can be identified in small numbers in the germinal centers of paraffin-embedded tissue sections of reactive tonsils (red arrow PRDM1-positive cells, blue arrow PAX5positive cells, purple arrow cells double positive for PRDM1 and PAX5)
PAX5 were not found in the reactive tonsils. This finding suggests that while identification of PRDM1+ PAX5+ cells may be physiologically normal, frequent presence of CD138+ PAX5+ cells is likely to be aberrant.

\section{Discussion}

The molecular mechanisms by which plasma cell differentiation is initiated and controlled is regulated by a panel of transcription factors and is an active field for study $[4,5,10$, 20]. Though PRDM1/Blimp-1 has long been considered to be the master regulator in plasma cell differentiation, additional studies have suggested that downregulated PAX5, instead of upregulated PRDM1/Blimp-1, is the initiating event in plasma cell differentiation [3]. Post-germinal center B cells serve as the precursors for plasma cells and memory B cells and have long been considered the putative cell of origin for LPL [21]. In the current model, the repression of PAX5 may play a critical role in determining the fate of post-germinal center B cells since memory B cells maintain high PAX5 expression.

The major goal of our study was to examine plasma cell differentiation in LPL by studying PAX5 expression in plasmacytic cells to determine if there is an abnormal PAX5 expression pattern and how it compares to MZL. In our study, we evaluated PAX5 coexpression in PRDM1-positive cells and CD138-positive cells. The use of both PRDM1 and CD138 to highlight plasma cells enabled us to follow PAX5 expression systematically along the plasma cell differentiation pathway, since PRDM1 expression precedes CD138. The percentages of PRDM1-positive and CD138-positive cells coexpressing PAX5 were both higher in LPL than bystander reactive plasma cells. In addition, the proportion of LPL cases showing PAX5 positivity in PRDM1-positive or CD138positive cells is significantly higher compared with MZL without plasmacytic differentiation. These findings suggest the hypothesis that PAX5 expression appears abnormally prolonged during plasma cell differentiation of the neoplastic B cells in LPL. In support of our findings, upregulated PAX5 expression in LPL but not multiple myeloma has also been reported through gene expression studies [22]. Comparison between LPL and MZL with plasmacytic differentiation revealed a higher percentage of CD138+ PAX5+ cells in LPL with marginal statistical significance, but no significant difference in the percentage of PRDM1+ cells with PAX5 positivity. In addition, a significantly higher proportion of LPLs showed PAX5 positivity in CD138+ cells compared to MZL with plasmacytic differentiation. These findings suggest that aberrantly sustained PAX5 expression to the fully mature plasma cell stage (i.e., CD138+) is a frequent occurrence in LPL but not MZL with plasmacytic differentiation. While PRDM1/PAX5 double staining may not be sufficiently specific to distinguish between LPL and MZL with plasmacytic differentiation, 
CD138/PAX5 staining, if present, particularly when it is over $5 \%$ of the plasma cells, is in favor of the diagnosis of LPL.

We have few cases that cannot be definitively placed in either category (MZL with plasmacytic differentiation vs LPL) based on clinical, morphologic, and immunophenotypic data. MYD88 mutation data is not available for these cases to allow more definitive subclassification. Comparative analysis of this very small group with LPL and other cases of MZL with plasmacytic differentiation revealed no significant difference in the results of double immunostaining but a conclusive interpretation is precluded by the small sample size. Further studies using the PAX5/CD138 double staining on a larger cohort may help determine whether cases with extramedullary disease and high levels of $\operatorname{IgM}$ paraprotein are biologically more akin to LPL or MZL.

The presence of weakly stained PRDM1+ PAX5+ plasmacytic cells in BM was identified in a minority of cases of MZL without plasmacytic differentiation in our study. Interestingly, the existence of rare B cells double positive for PRDM1 and PAX5 in the germinal center has been described [19]. Given our current understanding of the interplay between PRDM1 and PAX5 during terminal B cell differentiation, these PRDM1+ PAX5+ cells likely represent a transition stage from preplasmablasts to plasmablasts, in which PRDM1 is just induced and has not yet completely repressed PAX5. In addition, CD138+ PAX5+ cells can also be detected in MZL without plasmacytic differentiation in a subset of cases at a low percentage (up to $1.3 \%$ ). Similar cells were also reported to be present at low frequency in a subset of normal tissues (marrows and/or tonsils) [23]. These findings suggest that the frequent occurrence of aberrant PAX5 expression in CD138-positive plasmacytic cells in LPL may represent a pathological deregulation of a normal physiological process. Further studies are necessary for elucidating the clinical and biological significance of PRDM1+ PAX5+ and CD138+ PAX5+ cells in normal marrows.

Aberrant PAX5 expression in CD138-positive cells in LPL have been described in a case series study (published very recently during preparation of the current manuscript) with smaller sample size composed of BM and non-BM samples involved by LPL, plasma cell neoplasms, and MZL [23]. In that study, a significantly higher percentage of CD138+ plasma cells were positive for PAX5 in LPL compared to benign tissues and MZL, consistent with the findings in our study. In their study, six MZL lesions (four of six were BM specimens), all of which demonstrated monotypic plasma cell differentiation, exhibited very little aberrant PAX5 expression in CD138-positive plasma cells. This finding is also in line with our observations. However, our study has a larger number of MZL cases with plasmacytic differentiation and we included only BM specimens to eliminate potential confounding variables due to different tissue types and to facilitate the identification of distinguishing features in LPL that would help differentiate it from MZL in BM where a definitive diagnosis can be most difficult. Our study also included double staining for PRDM1 and PAX5, allowing us to trace PAX5 expression along the plasma cell differentiation pathway. Because of the use of both PRDM1/PAX5 and CD138/PAX5 double stains, we can conclude that PAX5 expression is abnormally sustained during plasma cell differentiation in LPL when we observe abnormal PAX5 staining in a subset of PRDM1+ cells and in a subset of CD138+ cells. Such a conclusion would not have been possible with CD138/PAX5 staining alone, as the possibility that PAX5-positive B cells ectopically express CD138 cannot be ruled out. Finally, with the inclusion of both MZL with or without plasmacytic differentiation in our study, the comparison among the neoplastic plasma cells in LPL, the neoplastic plasma cells in MZL, and the reactive bystander plasma cells in the marrow can be directly made.

Interestingly, only a subset of plasma cells in LPL showed aberrant PAX5 coexpression in both our study and in the previously described report [23]. One possibility is that PAX5 expression in the neoplastic plasma cells in LPL is upregulated at different levels. The sensitivity of double immunohistochemistry may result in preferential detection of those plasmacytic cells with higher PAX5 expression and thus may underestimate the percentage of plasma cells coexpressing PAX5. An alternative explanation is that aberrant PAX5 co-expression in plasma cells may be a subclonal phenomenon.

In summary, our findings in LPL suggest that altered plasma cell differentiation characterized by abnormally sustained PAX5 expression in a subset of plasmacytic cells is a frequent feature of this disease entity. This feature can also be seen, albeit to a lesser extent, in MZL. It is not known how this feature can contribute to the pathogenesis of LPL, but conceivably, abnormal PAX5 expression in these cells may disrupt the normal terminal Bcell differentiation process and reduce apoptosis normally associated with plasma cell differentiation. Further studies to elucidate the underlying mechanism for the aberrant PAX5 expression would help uncover the pathogenetic mechanisms of LPL.

Conflict of interest The authors declare that they have no conflict of interest.

\section{References}

1. Swerdlow SH et al (2008) WHO classification of tumours of haematopoietic and lymphoid tissues, Fourth Edition

2. Jourdan M et al (2009) An in vitro model of differentiation of memory B cells into plasmablasts and plasma cells including detailed phenotypic and molecular characterization. Blood 114(25):51735181

3. Kallies A et al (2007) Initiation of plasma-cell differentiation is independent of the transcription factor Blimp-1. Immunity 26(5):555-566

4. Shaffer AL et al (2002) Blimp-1 orchestrates plasma cell differentiation by extinguishing the mature $B$ cell gene expression program. Immunity 17(1):51-62 
5. Klein U et al (2006) Transcription factor IRF4 controls plasma cell differentiation and class-switch recombination. Nat Immunol 7(7):773-782

6. Reimold AM et al (2001) Plasma cell differentiation requires the transcription factor XBP-1. Nature 412(6844):300-307

7. Schebesta A et al (2007) Transcription factor Pax 5 activates the chromatin of key genes involved in B cell signaling, adhesion, migration, and immune function. Immunity 27(1):49-63

8. Delogu A et al (2006) Gene repression by Pax 5 in B cells is essential for blood cell homeostasis and is reversed in plasma cells. Immunity 24(3):269-281

9. Lin KI et al (2002) Blimp-1-dependent repression of Pax-5 is required for differentiation of B cells to immunoglobulin M-secreting plasma cells. Mol Cell Biol 22(13):4771-4780

10. Klein U, Dalla-Favera R (2007) Unexpected steps in plasma-cell differentiation. Immunity 26(5):543-544

11. Treon SP et al (2012) MYD88 L265P somatic mutation in Waldenstrom's macroglobulinemia. N Engl J Med 367(9):826833

12. Lampert IA, Van Noorden S, Wotherspoon AC (2005) Centrocytoid plasma cells of the germinal center. Appl Immunohistochem Mol Morphol 13(2):124-131

13. Schuster SR et al (2010) IgM multiple myeloma: disease definition, prognosis, and differentiation from Waldenstrom's macroglobulinemia. Am J Hematol 85(11):853-855

14. Feyler $\mathrm{S}$ et al (2008) IgM myeloma: a rare entity characterized by a CD20-CD56-CD117-immunophenotype and the $\mathrm{t}(11 ; 14) . \mathrm{Br} \mathrm{J}$ Haematol 140(5):547-551
15. Robillard N et al (2003) CD20 is associated with a small mature plasma cell morphology and $\mathrm{t}(11 ; 14)$ in multiple myeloma. Blood 102(3):1070-1071

16. Fonseca R et al (2002) Myeloma and the $\mathrm{t}(11 ; 14)(\mathrm{q} 13 ; \mathrm{q} 32)$; evidence for a biologically defined unique subset of patients. Blood 99(10):37353741

17. Hoyer JD et al $(2000)$ The $(11 ; 14)(\mathrm{q} 13 ; \mathrm{q} 32)$ translocation in multiple myeloma. A morphologic and immunohistochemical study. Am J Clin Pathol 113(6):831-837

18. Lin P et al (2004) Expression of PAX5 in CD20-positive multiple myeloma assessed by immunohistochemistry and oligonucleotide microarray. Mod Pathol 17(10):1217-1222

19. Cattoretti $\mathrm{G}$ et al (2005) PRDM1/Blimp-1 is expressed in human Blymphocytes committed to the plasma cell lineage. J Pathol 206(1):7686

20. Shaffer AL et al (2004) XBP1, downstream of Blimp-1, expands the secretory apparatus and other organelles, and increases protein synthesis in plasma cell differentiation. Immunity 21(1):81-93

21. Shaffer AL, Rosenwald A, Staudt LM (2002) Lymphoid malignancies: the dark side of B-cell differentiation. Nat Rev Immunol 2(12):920-932

22. Gutierrez NC et al (2007) Gene expression profiling of B lymphocytes and plasma cells from Waldenstrom's macroglobulinemia: comparison with expression patterns of the same cell counterparts from chronic lymphocytic leukemia, multiple myeloma and normal individuals. Leukemia 21(3):541-549

23. Roberts RMJ et al (2013) Nuclear protein dysregulation in lymphoplasmacytic lymphoma/waldenstrom macroglobulinemia. Am J Clin Pathol 139(2):210-219 